\title{
The exemplars of a strong whole were rated as more similar than were the exemplars of a weak whole
}

\author{
DONALD L. KING \\ Howard University, Washington, DC
}

\begin{abstract}
Exemplars of a diamond dot prototype and exemplars of an irregular dot prototype were formed by displacing dots according to the same probabilistic rule. The diamond exemplars were rated as much more similar to each other than were the irregular exemplars, even though the average dot displacement was equal for the two prototypes. The exemplars of each prototype may have formed a whole that corresponded to their prototype. Phenomenally, the diamond prototype forms a stronger whole than does the irregular prototype. Therefore, the whole formed by the exemplars of the diamond prototype should have been stronger. Consequently, the obtained result (along with other results) supports unit-similarity theory, which states that the perceived similarity among the components of a psychological unit, either a category or a perceptual whole (gestalt), is positively associated with the strength of the unit.
\end{abstract}

Stimuli from one category increase the perceived similarity of stimuli from a second category, according to rating and "same"'-"different" measures (King \& Atef-vahid, 1986; Sjoberg, 1972; Tversky, 1977). An illustrative result is that the inclusion of pairs of both American and European countries in the set of pairs that were rated for similarity increased the rated similarity of both types of pairs (Tversky, 1977). This result is accounted for by the theory that the perceived similarity between stimuli is positively associated with the strength of a unitlike phenomenal entity to which the stimuli belong (contribute).

Application of this unit-similarity theory assumes that the stimuli from one defined category increase the strength of a psychological category (unit) formed by the stimuli from a second defined category. The increase in the rated similarity of the stimuli from the second category then follows from the theory. For example, on phenomenal grounds, Brazil and Uruguay, the names of South American countries, seem to increase the extent to which Italy and Switzerland, the names of European countries, result in the category of European countries and vice versa. The increase in the rated similairty of Brazil and Uruguay and of Italy and Switzerland then follows from the theory. Tversky's (1977) explanation of the ability of stimuli from one category to increase the rated similarity of stimuli from a second category is a specific type of unit-similarity theory.

Unit-similarity theory also accounts for the contrasting

This research was supported by National Institute of Mental Health Grant 5-T-34-MH16580-03. The experiment was reported at the Eastern Psychological Association meeting in Baltimore, April 1982. Please send requests for reprints to Donald L. King, Department of Psychology, Howard University, C. B. Powell Building, 525 Bryant St., N.W., Washington, DC 20059. result that a stimulus that is more similar to two target stimuli than these two stimuli are to each other increases the rated similarity of the target stimuli (King, 1986). The assumption is that the more similar stimulus increases the strength of the category (unit) to which the target stimuli contribute and that the more similar stimulus also belongs to this category. The increase in the rated similarity of the target stimuli then follows from the theory. An illustrative finding is that pants both increased the rated similarity of gloves and purse and was rated as more similar to gloves and purse than gloves and purse were to each other. For this finding, unit-similarity theory assumes that pants also increased the strength of the category of CLOTHING and that gloves, purse, and pants all belonged to this category. Because purse and pants presumably belonged to a stronger category, the increase in their rated similarity is accounted for. The ability of stimuli that are more similar to two target stimuli then these stimuli are to each other to increase the rated similarity of the target stimuli also opposes the distance-density theory (Krumhansl, 1978), because the inclusion of more similar contextual stimuli should increase density and hence decrease perceived similarity.

A large range of stimuli or an extreme stimulus impairs the absolute identification of intermediate and/or more similar stimuli on the same dimension (Gravetter \& Lockhead, 1973; Pollack, 1952). This anchor-range result resembles the finding that stimuli from one category increase the rated similarity of stimuli from a second category. This is because category rating (Bevan \& Pritchard, 1963), magnitude estimation (DiLollo \& Kirkham, 1969), and "same"-“different" results (King, 1983) argue that the impairment in absolute identification is due to an increase in perceived similarity. Unit-similarity theory explains the anchor-range 
result in the same way, namely, with the assumption that dissimilar stimuli increase the strength of the category formed by the more similar stimuli. For example, the very low-intensity sound used by Gravetter and Lockhead (1973) may have increased the strength of a category of loud sounds resulting from the two high-intensity sounds as well as the perceived similarity of the high-intensity sounds. The increase in the perceived similarity of the loud sounds would then explain the decrease in the ability to identify them. Furthermore, memorial and judgmental interpretations of the anchor-range effect face serious difficulties (King \& Atef-vahid, 1986).

The unit-similarity theory applies to perceptual units (wholes, gestalts) as well as to categories. One gestalt result is that the parts of single phenomenal wholes are perceived as closer together (i.e., in more similar spatial positions) than the same parts when they are members of different phenomenal wholes (Coren \& Girgus, 1980). A second gestalt result is that the perception of a spread of color tends to be confined to the parts of the same phenomenal whole (e.g., Day, 1983). Unit-similarity theory also applies to results that imply that perceived similarity is causal, for example, the result that monocular viewing increases both the perceived similarity in spatial position of adjacent horizontal spheres and the frequency with which the spheres form apparent rows (Rock \& Brosgole, 1964). In all of these studies, parts are perceived as more similar when they form a stronger phenomenal whole, thus supporting unit-similarity theory.

Work with figural stimuli has relied on measures of the perception of specific attributes (either spatial position or color). However, evidence that the anchor-range effect occurs with figural stimuli was recently obtained with the "same"-"different" measure of perceived similarity (King \& Atef-vahid, 1986). The present experiment also sought to test unit-similarity theory using figural stimuli and a measure of overall similarity, in this case ratings of similarity.

The stimuli were exemplars of dot pattern prototypes that formed strong and weak wholes. Exemplars of a dot pattern prototype can result in the recognition and retention of the prototype, even though the exemplars are fairly dissimilar to the prototype (Posner \& Keele, 1970). Consequently, the assumption was that exemplars of the same prototype tend to form a unit-like entity that corresponds to their prototype. Given this assumption, the exemplars of the strong whole prototype should result in a stronger unit-like event than should the exemplars of the weak whole prototype. Unit-similarity theory maintains that the exemplars that contribute to the stronger unit should be perceived as more similar. Therefore, this theory predicts that the exemplars of the strong prototype will be rated as more similar than the exemplars of the weak prototype. This prediction is also of interest because it involves a new way of comparing strong and weak wholes.

The number of exemplars of a prototype was also varied, because there are indications that the strength of a psychological category increases with the number of ex- emplars that belong to the corresponding experimenterdefined category (Homa, Rhoads, \& Chambliss, 1979).

\section{Stimuli \\ METHOD}

Figure 1 shows the diamond and irregular dot pattern prototypes that were used. Phenomenally, the diamond pattern results in a stronger whole than does the irregular pattern.

The prototypes were formed with the help of a $38 \times 38$ grid. Each corner of the diamond was positioned 5 grid squares away from the grid's edge. The positions of the dots for the irregular pattern were determined randomly.

The same probabilistic rule was used to generate each exemplar of the two prototypes. It resembles the one used by Posner, Goldsmith, and Welton (1967). However, the present rule resulted in exemplars that more nearly matched their prototypes than did the Posner et al. rule. Each dot of each prototype was not moved, was moved to one of the grid squares that formed a ring around the dot, was moved to one of the 16 squares of the next closest ring, or was moved to one of the 24 squares of the next ring removed. The probabilities of these moves were $.4, .3, .2$, and .1 in the order of least to greatest distance moved. The specific grid square in a ring to which a dot was assigned was also determined randomly.

Exemplars of the same prototype appeared in a column on the same page, as described below. The size of each exemplar was $4.1 \times 4.1 \mathrm{~cm}$.

\section{Design, Procedures, and Subjects}

In total, 48 exemplars were generated for each prototype. The first four exemplars comprised one set of A, B, C, and D items (Exemplar Set 1-4), the second four exemplars comprised a second set of these items (Exemplar Set 5-8), and so forth, until 12 sets of exemplars for each prototype were obtained. An AB group rated the first two (A and B) exemplars of Exemplar Set 1-4, a second AB group rated the first two exemplars of Exemplar Set 5-8, and so forth. This was done for both the diamond and irregular patterns. Thus, there were 12 independent $\mathrm{AB}$ groups, and each group rated one diamond and one irregular pair of exemplars. There were also 12 ABC groups. These groups received $A B, A C$, and $B C$ pairs to rate (e.g., the first three exemplars [A, B, and C] of Exemplar Set 1-4 were used for the first of these groups). There were also $12 \mathrm{ABCD}$ groups. These groups received $\mathrm{AB}$, AC, AD, BC, BD, and CD pairs to rate. All four exemplars of a set were used for these groups. Therefore, there were $12 \times 3$, or 36 , independent groups. Twelve sets of exemplars were used, because with this relatively large number, the average displacement of the exemplar dots from their positions in the prototypes should be essentially identical for the two prototypes.

As stated above, all the exemplars of the same prototype appeared on the same page. Therefore, one (AB), three (AB, AC, and $\mathrm{BC})$, or six (AB, AC, AD, BC, BD, and $C D)$ such pairs appeared in a column, and there were two such pages, one for the exemplars of each prototype. One half of the subjects of each of the 36 groups received the page of diamond exemplars first, and the other half received the page of irregular exemplars first.

A line appeared to the right of each pair of exemplars. It was calibrated from 1 to 9 , and subjects marked it to indicate their similarity ratings.
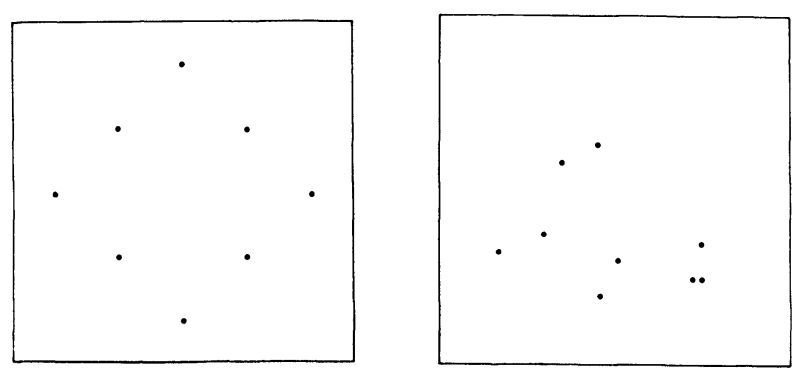

Figure 1. The diamond and irregular dot prototypes. 
An initial page contained instructions and an illustrative pair and line. The instructions did not mention the type of attribute to be used for rating similarity.

There were 2 subjects in each of the 36 groups. The subjects were tested in small groups. They were students enrolled in the introductory psychology classes at Howard University. Participation helped to fulfill the course requirements.

\section{RESULTS}

The similarity ratings of the pairs of exemplars that all groups received (i.e., the $\mathrm{AB}$ pairs) were analyzed. The analysis of variance (ANOVA) was a 12 (exemplar set: $1-4,5-8$, etc.) $\times 3$ (number of pairs on a page: 1,3 , and 6) $\times 2$ (prototype: diamond and irregular) factorial. Prototype was a within-subject factor.

The main effect of the prototype factor was significant; the mean similarity ratings for the diamond and irregular target pairs were 6.3 and 3.3 , respectively $[F(1,36)=$ $77.47, p<.001]$. The interaction between the number of pairs and prototype factors was also significant $[F(2,36)=3.53, p<.05]$. Groups that received 1,3 , and 6 pairs on a page rated the diamond exemplar pairs comparably, the means equaling $6.5,6.2$, and 6.3 , respectively. Groups that received 1 and 3 pairs on a page also rated the irregular exemplar pairs comparably, the means equaling 3.9 and 4.1 , respectively. However, the irregular exemplar mean for the groups that received six pairs on a page was only 2.1 .

\section{DISCUSSION}

Pairs of exemplars formed from a diamond dot prototype were rated as much more similar than were pairs of exemplars formed from an irregular dot prototype. This result occurred even though the average distance between a dot in the exemplars and the corresponding dot in the prototype must have been essentially identical for the two prototypes. Two or more exemplars of each prototype may have resulted in the apprehension of a whole (gestalt) that corresponded to the prototype. In support of this possibility, exemplars of dot prototypes result in the recognition and retention of their prototypes (Posner \& Keele, 1970). Phenomenally, the diamond prototype forms a stronger whole than does the irregular prototype. It follows that the diamond exemplars contributed to a stronger whole than did the irregular exemplars. Unit-similarity theory maintains that the perceived similarity between components increases with the strength of the unit-like entity to which they contribute. The exemplars correspond to components, and a whole is a unit-like entity. Consequently, unit-similarity theory accounts for the result that the diamond exemplars were rated as more similar than the irregular exemplars.

A second result was that groups that received six pairs on a page rated the irregular exemplar pairs as lower in similarity than did groups that received one or three pairs on a page. This result was unexpected. This result is interesting, because, as far as I know, there have been no other reports that the addition of contextual stimuli decreases the rated similarity of target stimuli. The decrease seems difficult to understand, however, because it occurred for only the irregular exemplars.

Strong and weak phenomenal wholes have been compared in other ways. The introduction mentioned research on the perceived similarity of components of wholes. In addition, variation in a logically irrelevant component of a whole hurts classification performance more for strong wholes than for weak wholes (Pomerantz \& Schwaitzberg, 1975). Also, objects are more poorly identified when they are part of wholes than when they are single objects (Prinzmetal \& Banks, 1977). Therefore, the general expectation is that as the strength of a phenomenal whole increases, then the rated similarity of exemplars of this whole increases, the perceived similarity of components of this whole increases, the in terference with classification performance produced by irrelevant variation in components of this whole increases, and the ability to identify components of this whole decreases.

Unfortunately, the result that pairs of diamond exemplars were rated as more similar than were pairs of irregular exemplars may not be general. First, only two prototypes were used. Second, the two prototypes differed in at least one other important respect (i.e., the dots of the diamond prototype were farther apart than the dots of the irregular prototype). Clearly, a variety of dot prototypes that form strong and weak wholes might be used to determine if the present results are general.

In conclusion, exemplars of a diamond dot prototype, a strong whole, were rated as more similar than were exemplars of an irregular dot prototype, a weak whole, even though both types of exemplars were generated in the same way. This finding supports the theory that the perceived similarity between stimuli increases with an increase in the strength of the unit-like entity to which they contribute.

\section{REFERENCES}

Bevan, W., \& Pritchard, J. F. (1963). The anchor effect and the problem of relevance in the judgment of shape. Journal of General Psychology, 69, 147-161.

CoRen, S., \& Girgus, J. S. (1980). Principles of perceptual organization and spatial distortion: The gestalt illusions. Joumal of Experimental Psychology: Human Perception \& Performance, 6, 404-412.

DAY, R. H. (1983). Neon color spreading, partially delineated contours, and the formation of illusory contours. Perception \& Psychophysics, 34, 488-490.

DiLollo, V., \& KirKham, R. (1969). Judgmental contrast effects in relation to range of stimulus values. Journal of Experimental Psychology, 81, 421-427.

GraveTter, F., \& LockHeAd, G. R. (1973). Criterial range as a frame of reference for stimulus judgment. Psychological Review, 80, 203-216.

Homa, D., RhoAds, D., \& Chambliss, D. (1979). Evolution of conceptual structure. Journal of Experimental Psychology: Human Learm ing \& Memory, 5, 11-23.

KING, D. L. (1983). Anchor research: Evidence for an increase in the perceived similarity of stimuli obtained with a speeded response paradigm. Journal of Experimental Psychology: Human Perception \& Performance, 9, 474-486.

KING, D. L. (1986). Similar as well as dissimilar stimuli increase rated similarity. Manuscript submitted for publication.

KING, D. L., \& ATEF-VAHID, M. (1986). Two extensions of the anchorrange effect. Perception \& Psychophysics, 39, 96-104.

KrUmHANSL, C. L. (1978). Concerning the applicability of geometric models to similarity data: The interrelationship between similarity and spatial density. Psychological Review, 85, 445-463.

Pollack, I. (1952). The information of elementary auditory displays. Journal of the Acoustical Society of America, 24, 745-749.

Pomerantz, J. R., \& SCHWATtZBerg, S. D. (1975). Grouping by proximity: Selective attention measures. Perception \& Psychophysics, 18, 355-361.

Posner, M. I., Goldsmith, R., \& Welton, K. E., JR. (1967). Perceived distance and the classification of distorted patterns. Journal of Experimental Psychology, 73, 28-38.

Posner, M. I., KeELe, S. W. (1970). Retention of abstract ideas. Journal of Experimental Psychology, 83, 304-308.

Prinzmetal, W., \& BANKs, W. P. (1977). Good continuation affects visual detection. Perception \& Psychophysics, 21, 389-395.

RoCK, I., \& BrosGole, L. (1964). Grouping based on phenomenal proximity. Journal of Experimental Psychology, 67, 531-538.

Sjoberg, L. (1972). A cognitive theory of similarity. Goteborg Psychological Reports, 2(No. 10).

TVErSKY, A. (1977). Features of similarity. Psychological Review, 84, 327-352.

(Manuscript received for publication August 25, 1986.) 